\title{
Why does the effect of short-SOA exogenous cuing on simple RT depend on the number of display locations?
}

\author{
J. TOBY MORdKOFF ANd RoSE HALTERMAN \\ University of Iowa, Iowa City, Iowa \\ AND \\ Peggy Chen \\ University of Medicine and Dentistry of New Jersey, Newark, New Jersey \\ and Kessler Medical Rehabilitation Research and Education Center, West Orange, New Jersey
}

\begin{abstract}
When the stimulus onset asynchrony (SOA) between the cue and target is short (i.e., less than $200 \mathrm{msec}$ ) and the number of display locations is small (e.g., only two), exogenous spatial cues produce a benefit in simple response time (RT). However, several recent experiments have found significant costs in these tasks when a large number of display locations is employed (e.g., eight), even at the very short SOAs that usually produce a benefit. The present study explored the dependence of exogenous cuing on the number of display locations and found evidence that both the overall validity of the cues and the specific validity of the cue on the previous trial have strong, additive effects. When a large number of display locations is used, both of these factors work against a benefit of exogenous cuing on simple RT, reversing the typical finding into a cost. These two effects are suggested to occur within motor and perceptual processes, respectively.
\end{abstract}

In the exogenous-cuing paradigm (e.g., Posner \& Cohen, 1984), a noninformative spatial cue precedes the target by a short delay (i.e., a stimulus onset asynchrony [SOA] of less than $200 \mathrm{msec})$. On a typical trial in such an experiment, one of several placeholders briefly changes in luminance, acting as the cue, and is closely followed by a target appearing within one of the placeholders. The "standard" result from this task is a benefit in simple response time (RT) when the cue is presented in the same location as the subsequent target (valid-cue trials), as opposed to some other location (invalid-cue trials). The specific magnitude of the cuing effect varies as a function of several stimulus factors (see, e.g., Maruff, Yucel, Danckert, Stuart, \& Currie, 1999; McAuliffe \& Pratt, 2005; Pratt, Hillis, \& Gold, 2001), but is almost always positive and reliable when only two display locations are employed.

Recently, however, several experiments have found large, negative effects of short-SOA exogenous cuing on simple RT (i.e., a cost on valid-cue trials) when the number of display locations is much greater than two (e.g., Chen, Moore, \& Mordkoff, 2008; Gawryszewski, Carreiro, \& Magalhães, 2005; see also Tassinari, Aglioti, Chelazzi, Peru, \& Berlucchi, 1994, Experiments 3 and 4 for an earlier example). These findings are important, because they raise questions about the ability of such cues to capture attention automatically; most explicit models of attentional capture by exogenous cues (e.g., Theeuwes, 2004; Yantis, 1996) would always predict a benefit. Although some recent work has stressed the strategic nature of attentional cuing (e.g., Folk, Remington, \& Johnston, 1992; Lupiáñez, Milliken, Solano, Weaver, \& Tipper, 2001), it is not yet clear why merely increasing the number of display locations would cause the effect of an uninformative spatial cue to reverse from being a benefit to becoming a cost.

To see why the number of display locations might have this effect, consider two exogenous-cuing experiments, one using two display locations and the other using four. Besides the obvious difference in the number of places where cues and targets are presented, there are at least two other differences. First, when the display size is changed from two to four, the probability of the target's appearing at the same location as the cue drops from $50 \%$ to $25 \%$ (i.e., the overall validity of the cues is reduced), because the locations of the cue and target are both selected at random for exogenous cuing. Second, and directly following from the first, the probability of the previous trial involving a valid cue also drops from $50 \%$ to $25 \%$. Either or both of these changes could be responsible for the change in the observed cuing effect. The overall validity of the cues could determine whether participants allow their attention to be captured by a cue (or maybe even provide a motive for them to actively resist attentional capture; see Rem- 
ington, Johnston, \& Yantis, 1992). Alternatively, it could affect how the system is configured, in such a way that overall validity could determine whether the activations extracted from the cue and target are allowed to coactivate the overt response (see Mordkoff, Miller, \& Roch, 1996). Similarly, the validity of the cue on the previous trial could also have a modulating effect on attentional capture (see Frith \& Done, 1986, for a related idea), with the likelihood of capture being partly determined by whether capture was helpful on the preceding trial. Finally, the validity of the previous cue could be used to determine whether cues and targets coactivate responses.

Previous studies have examined the changes in the effects of exogenous cues as a function of display size and the validity of the cue on the previous trial. Pratt, Adam, and McAuliffe (1998), for example, found decreases in the negative cuing effect that is observed at long SOAs as display size increased. More recently, Dodd and Pratt (2007) have shown that the long-SOA negative cuing effect (usually referred to as inhibition of return) is larger on trials that follow invalid cues. However, all of this work has concerned situations where negative cuing effects are the norm. The specific goal of the present research was to uncover the causes of the negative cuing effects that can be observed with short SOAs when large displays are employed.

\section{The Present Experiment}

The present experiment included conditions with two, three, or four display locations, and the cuing effects were analyzed in terms of both this factor and whether the previous trial involved a valid or invalid cue. In all cases, the locations of the cue and target were selected at random; therefore, the manipulation of the number of display locations was also a manipulation of the overall probability that the cue would be valid. Likewise, because the trials were run in a random order, some trials were preceded by valid-cue trials and others were preceded by invalid-cue trials. Thus, this experiment allows for separate tests of the effect of overall cue validity and of the effect of the validity of the cue on the previous trial, as well as any possible interaction between the two.

\section{METHOD}

\section{Participants}

A total of 48 undergraduates participated in fulfillment of a course requirement, with 16 participating in each of the three groups (defined by the number of display locations). All of the participants provided informed consent but were naive as to the study's design and purpose. Each participant was tested in a single session lasting less than $1 \mathrm{~h}$.

\section{Stimuli and Procedure}

Each trial began with $1,000 \mathrm{msec}$ of fixation, surrounded by 2 , 3 , or 4 empty-square placeholders. The fixation cross subtended $0.65^{\circ}$ of visual angle; each placeholder was $1.95^{\circ}$ and appeared $6.20^{\circ}$ from fixation. The "clock" position of one of the placeholders was selected at random on every trial; the other placeholders were then spaced evenly on an imaginary circle around fixation. At the end of the fixation period, one placeholder brightened for $100 \mathrm{msec}$. On a majority of the trials $(\sim 89 \%)$, a $1.12^{\circ}$ filled-square target was presented inside one of the placeholders, appearing $50 \mathrm{msec}$ after the cue was brightened (i.e., the cue and target overlapped by $50 \mathrm{msec}$ ). The target remained visible until a response was made or $1,000 \mathrm{msec}$ had elapsed. On the remaining trials $(\sim 11 \%)$, no target was presented.

The participants' task was to respond by pressing a button with their favored index finger as soon as possible following the appearance of a target. The instructions requested that participants be as fast as possible yet maintain a high rate of accuracy. All errors (including false alarms, anticipations, and failures to respond within $1,000 \mathrm{msec}$ on target-present trials) were followed by a low tone (plus a text message during the first block of practice). Each block of trials included 32 target-present trials, 4 catch trials, and 3 randomly selected warm-up trials. A recovery trial was also inserted after an error, and the error trial was rerun at a random point later in the block. Given the rarity of errors, a majority of blocks included 39 or 41 trials and required less than 2 min. Participants performed 16 blocks, receiving summary feedback during an enforced 7 -sec break between adjacent blocks. The first 4 blocks were treated as practice and were not included in the analyses.

\section{RESULTS AND DISCUSSION}

The data from warm-up and recovery trials were excluded from the analysis, as were those from all trials that immediately followed a catch trial, because the validity of the previous cue was undefined. Before the main analysis was conducted, the assumptions of ANOVA were examined using the Box test of the covariance matrix and separate applications of Levene's test to each repeated measure. There were no significant violations (all $F \mathbf{s}<1, p \geq$ $.621)$, so the analyses proceeded as planned. The mean RTs (for correct responses only) and error rates are provided in Table 1.

The three-way ANOVA on the mean RTs did not find an interaction between display size, current cue validity, and previous cue validity $(F<1, p=.829)$. However, the two-way interaction between display size and current cue validity was reliable $\left[F(2,45)=4.33, p=.019 ; \eta^{2}=.16\right]$, as was the interaction between current cue validity and previous cue validity $\left[F(1,45)=16.33, p<.001 ; \eta^{2}=\right.$ .27]. There was also a main effect of previous cue validity $\left[F(1,45)=33.05, p<.001 ; \eta^{2}=.42\right]$, but notably, the main effect of current cue validity was not significant $[F(1,45)=1.54, p=.221]$. No other effects approached significance $($ all $F \mathrm{~S}<1)$.

To clarify these findings, the data were first reexamined in terms of the cuing effect (i.e., the difference between valid- and invalid-cue trials), instead of the mean RTs. This reanalysis is illustrated in Figure 1, which shows that there were main effects of both display size and previous cue validity (which correspond to the two-way interactions from the original ANOVA). In addition, this depiction makes it clear how the influences of display size and previous cue validity on the size of the cuing effect were almost perfectly additive (which corresponds to the lack of a three-way interaction in the analysis of the mean RTs). With regard to the interaction between display size and current cue validity, the apparent shift from a positive cuing effect for Display Size 2 to a negative cuing effect for Display Size 4 was verified by several follow-up tests. Collapsing across previous cue validity, the cuing effect for Display Size $2(19 \mathrm{msec})$ was larger than that for 
Table 1

Mean Response Times (in Milliseconds) and Error Rates (in Percent) As a Function of Display Size, Previous Cue Validity, and Current Cue Validity

\begin{tabular}{|c|c|c|c|c|c|c|c|c|c|}
\hline \multirow[b]{2}{*}{ Cue } & \multicolumn{3}{|c|}{2} & \multicolumn{3}{|c|}{3} & \multicolumn{3}{|c|}{4} \\
\hline & RT & $\%$ Miss & $\% \mathrm{FA}$ & RT & $\%$ Miss & $\% \mathrm{FA}$ & RT & $\%$ Miss & $\% \mathrm{FA}$ \\
\hline \multicolumn{10}{|c|}{ Valid Previous Trial } \\
\hline Valid & 280 & 1.3 & & 296 & 0.8 & & 288 & 0.3 & \\
\hline Invalid (adjacent) & & & & 307 & 1.0 & & 287 & 0.2 & \\
\hline Invalid (opposite) & 307 & 1.2 & & & & & 291 & 0.2 & \\
\hline & & & 12.3 & & & 10.7 & & & 12.8 \\
\hline \multicolumn{10}{|c|}{ Invalid Previous Trial } \\
\hline Valid & 298 & 1.2 & & 316 & 0.5 & & 310 & 0.6 & \\
\hline Invalid (adjacent) & & & & 314 & 0.4 & & 292 & 0.5 & \\
\hline Invalid (opposite) & 308 & 1.2 & & & & & 290 & 0.5 & \\
\hline & & & 7.5 & & & 7.7 & & & 10.0 \\
\hline
\end{tabular}

Display Size $4(-9 \mathrm{msec})(p=.005)$, whereas the cuing effect for Display Size 3 (4 msec) did not differ reliably from either extreme (for both, .10<p<.20). Furthermore, a trend analysis confirmed the apparent linearity of this shift from a positive to a negative cuing effect as display size increased $(p=.019)$. At the same time, the consistent influence of previous-trial validity on the size of the cuing effect was $17 \mathrm{msec}$.

The second follow-up analysis sought to explicate the two-way interaction between current cue validity and previous cue validity that was observed in the main analysis. This was done by collapsing the data across display size (as justified by the lack of a three-way interaction), which, in this case, corresponds to ignoring the fact that there were three different groups of participants. The results from this reanalysis are shown in Figure 2, which suggests that there was a significant simple main effect of previous cue validity when the current cue was valid ( $p<$ $.001)$, but that there was no such effect when the current cue was invalid ( $p=.091)$. Alternatively, one could also view these data as showing a simple main effect of current cue validity when the previous cue was valid ( $p=$ .003 ), but not when the previous cue was invalid ( $p=$ .430). Finally, a third way to look at these data is in terms of homogeneous subsets, which ignores that the four cells actually come from a $2 \times 2$ design; this analysis found mean RT to be lowest when two valid trials occurred in succession $(p=.021)$, whereas the three other conditions were statistically equivalent (all $p \mathrm{~s} \geq .147$ ). In short, the interaction between current cue validity and previous cue

Main Experiment

\section{Controls}

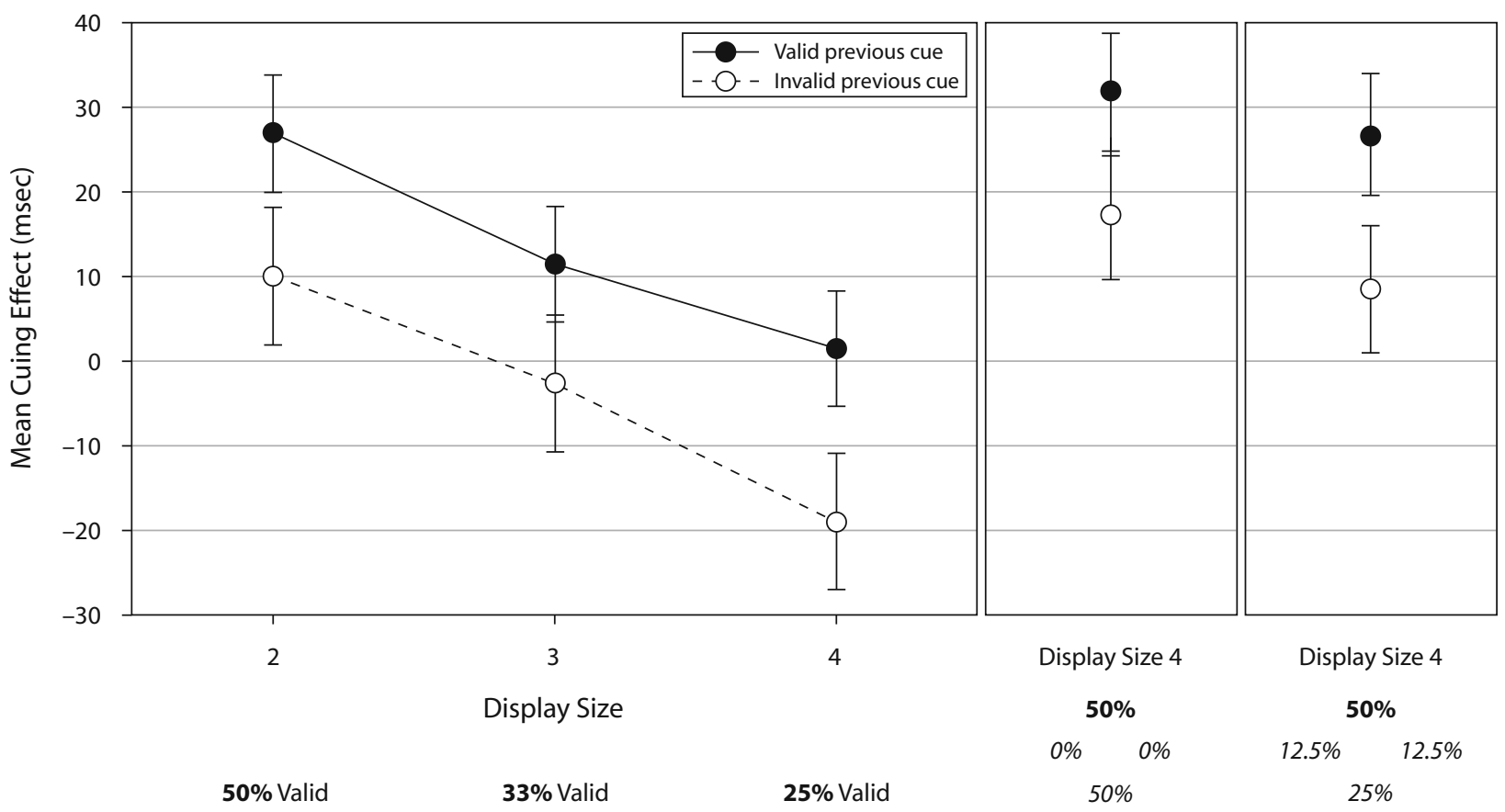

Figure 1. Mean cuing effect (i.e., invalid-cue RT minus valid-cue RT) as a function of the number of display locations and/or overall cue validity (percent valid in bold), as well as the validity of the cue on the previous trial. 


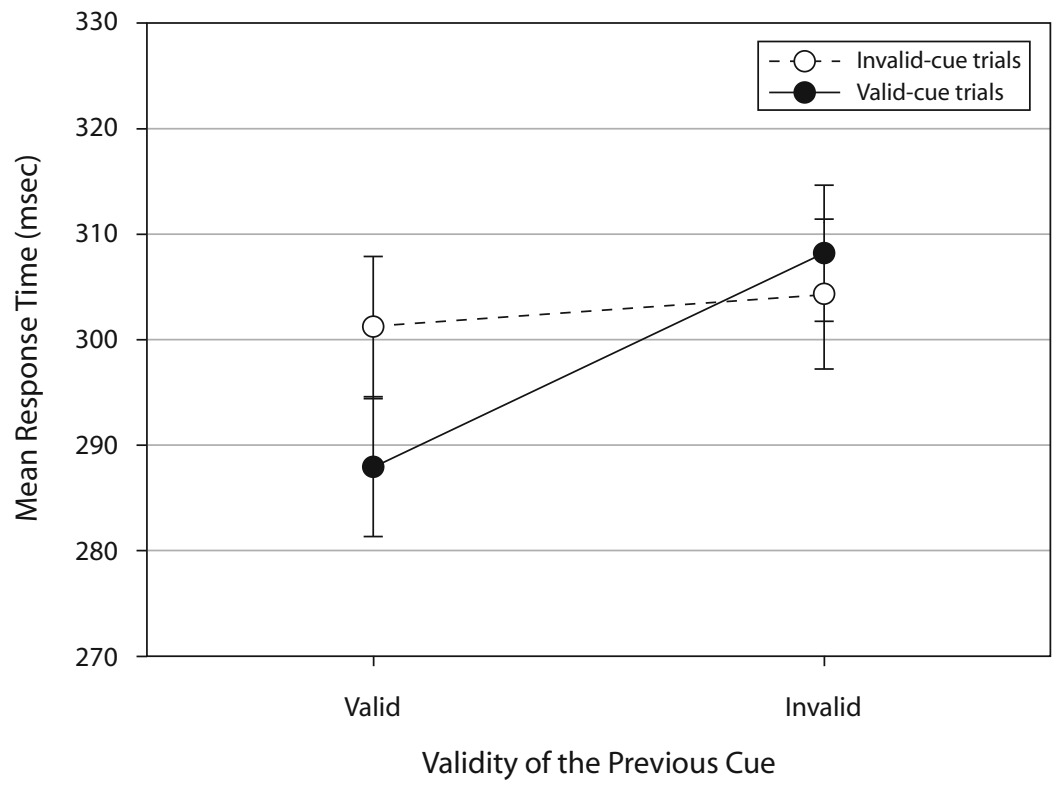

Figure 2. Mean response time as a function of the validity of the cue on the previous trial and current cue validity, collapsed across display size.

validity was of the type that is sometimes referred to as "completely underadditive."

Finally, it is also worth noting that, when more than three display locations are used, the target can be presented at various distances from the cue on invalid-cue trials (see, e.g., Chen et al., 2008). In the present experiment, whether the target appeared adjacent to or opposite from the cue had no effect on performance with Display Size 4, regardless of whether the previous cue was valid or invalid (see Table 1) (all $F \mathrm{~s}<1$ ). This is important, because it suggests that the present negative effects of exogenous cues at a short SOA could well be distinct from the negative effects that are often found at long SOAs. When long SOAs are used, an advantage for the opposite location over the adjacent locations has sometimes been observed (e.g., Pratt et al., 1998; but see also Posner \& Cohen, 1984).

With regard to accuracy, the participants missed an average of about $1 \%$ of the targets and made false alarm errors on approximately $10 \%$ of the catch trials (see Table 1). In the analysis of the error data, the only reliable effect was the difference in false alarm rates as a function of the validity of the cue on the previous trial $[F(1,45)=304.1$, $\left.p=.001 ; \eta^{2}=.24\right]$. A false alarm error was more likely to occur following a valid-cue trial (12\%) than following an invalid-cue trial $(8 \%)$. No other main effect or interaction approached significance (all $p \mathrm{~s} \geq .286$ ).

In summary, this experiment produced evidence in favor of both of the possible changes that were discussed in the introduction: (1) When display size is increased, the overall validity of the cues is reduced, which causes a reduction in the benefit of exogenous cuing, and (2) when display size is increased, the probability of the previous cue's being valid is reduced, which likewise causes a reduction in the benefit of exogenous cuing. These two fac- tors, working together, are sufficient to reverse the typical benefit of exogenous cuing (to being a cost) when display size reaches four. In other words, when exogenous cues are valid on only $25 \%$ of the trials, exogenous cuing incurs a significant cost.

\section{Control Tasks}

One question unanswered by the main experiment is whether the effect of the number of display locations is due to the change in the overall validity of the cues, as suggested in the introduction and as claimed in the previous paragraph. To address this issue, several control experiments have been conducted, two of which will be reported here in some detail. In both, each trial began in the same manner as was used for Display Size 4 in the main experiment: with four empty placeholders surrounding fixation, one of which was briefly brightened as the cue. In the first control, the target always appeared at the cued location or directly opposite, so that overall cue validity was $50 \%$, even though the number of display locations was four. This particular method had several advantages over others that were considered. Most of all, the average expected location of the target remained at the center of the screen. This made endogenous shifts of attention unlikely, given the evidence that such shifts cannot be aimed at two different locations at the same time (e.g., Posner, Snyder, \& Davidson, 1980). Thus, the overall validity of the cues in this control task was equal to that in the Display Size 2 condition of the main experiment (viz., 50\%), but this task involved four target locations. Sixteen new participants were recruited from the same pool as before.

The results from this control strongly support the idea that the overall validity of the cues, not the number of locations employed, was the critical factor (see Figure 1). The data were very similar to those from Display Size 2 
in the main experiment (i.e., there were no main effects or interactions in a post hoc ANOVA that included only these two sets of data (all $F \mathrm{~s}<1$ ). The cuing effect was $32 \mathrm{msec}$ following valid trials and $17 \mathrm{msec}$ following invalid trials (both being different from zero; $p<.05$ ). This demonstrates that benefits of exogenous cuing can be observed in simple-RT experiments involving four display locations, as long as the overall validity of the cues is $50 \%$.

Before we move on to the second control, it is important to note that the first control experiment was not a mere replication of the Display Size 2 condition from the main experiment. Although it is true that the target could appear only at one of two locations on a given trial, the specific two locations were not known to the subject until the cue appeared. Thus, to argue that this first control was the same as Display Size 2, one must assume that two of the four locations could be completely suppressed or otherwise ignored in $50 \mathrm{msec}$ or less, because this was the SOA between the cue and the target. Such an assumption appears to have been ruled out by several findings. Most of all, a $100 \%$ invalid peripheral cue (which therefore reduces the number of potential target locations) has, if anything, a detrimental effect on performance, even when presented as much as $150 \mathrm{msec}$ in advance (e.g., Folk et al., 1992); this is the opposite finding from what needs to be assumed. Similarly, invalid exogenous cues do not alter either the mean RT or the magnitude of the set-size effect in visual search (when compared with a neutral condition), even when these cues are presented approximately $100 \mathrm{msec}$ in advance (Carrasco \& Yeshurun, 1998). In summary, there is reason to believe that the effective number of display locations cannot be reduced in $50 \mathrm{msec}$ or less. Therefore, the results from the first control support the idea that it is the overall validity of the cues, and not the number of display locations, that determines the value of the cuing effect.

In the second control, the overall validity of the cues was $50 \%$, even though targets could appear at any of the four locations. On half of the trials, the target appeared at the cued location; on one quarter of the trials, it appeared at the opposite location; and on the remaining quarter of the trials, the target appeared at one of the adjacent locations, divided evenly between the two. Although this design introduced the possibility of a voluntary shift of attention and therefore the task was not an exogenous-cuing task by strict definition, this control was included because it clearly maintained a display size of four, while still having cues that were valid on $50 \%$ of the trials. Fortunately, previous work strongly suggested that an endogenous shift of attention was highly unlikely under these conditions, because voluntary shifts take much longer than the $50 \mathrm{msec}$ that were available in the present case (see, e.g., Luck, Hillyard, Mouloua, \& Hawkins, 1996; Müller \& Findlay, 1988).

The results from the second control also supported the idea that it is the overall validity of the cues that matters (see Figure 1). The cuing effects were 27 and $9 \mathrm{msec}$ following valid- and invalid-cue trials, respectively, which are virtually identical to (i.e., within $1 \mathrm{msec}$ of) the results from Display Size 2 in the main experiment. As was true for the first control experiment, an ANOVA that compared these data with those from Display Size 2 found no main effects or interactions (all $F_{\mathrm{s}}<1$ ).

\section{GENERAL DISCUSSION}

The present study was motivated by several recent findings of a significant cost of exogenous cuing when the number of display locations was high (e.g., Chen et al., 2008; Gawryszewski et al., 2005). The working hypothesis was that the decrease in the overall validity of the cues, as well as the decrease in the probability that the previous trial involved a valid cue, were jointly responsible for this change in the "standard" result (e.g., Posner \& Cohen, 1984). The present experiment provided some direct support for both of these ideas.

At the same time, however, the rather stunning additivity of these two effects (see Figure 1) places some very strong limits on how they ought to be interpreted. For example, it cannot be the case that the overall validity of the cues and the validity of the cue on the previous trial both act as multiplicative moderators or "gain" controls on how effectively the cue on the current trial will capture attention. Such a model would predict a statistical interaction between these two factors (i.e., different slopes for the two plots in Figure 1), with the effect of the number of display locations being smaller on trials that follow an invalid cue.

Instead, the additivity of these effects would seem to suggest the involvement of two separate mechanisms, possibly corresponding to the perceptual and motoric components of the original model of simple RT (e.g., Donders, 1868/1969; see also Boring, 1950, for some discussion of these two components; and see, e.g., Klein, 1980, for recent related ideas in the general context of spatial cuing). For example, the validity of the previous cue could help determine whether attention is allowed to be captured (perceptual), whereas the overall validity of the cues might determine whether the activations extracted from the cue and target are allowed to coactivate the current response (motoric). These ideas are consistent with other evidence concerning the role of strategic factors in attentional capture (e.g., Folk et al., 1992; Lupiáñez et al., 2001). They are also compatible with findings that response force increases when additional items are included in the same display as that in which a target is included (e.g., Giray \& Ulrich, 1993; Mordkoff et al., 1996).

It should be noted that the effects reported here are almost definitely limited to simple RT and do not generalize to tasks that require visual discriminations. There have been myriad demonstrations of positive cuing effects with cue validities as low as $25 \%$ (i.e., Display Size 4 ) when the task required a forced choice response (e.g., Folk et al., 1992). The reasons for this dependence on task remain unclear, but converge with two other, known differences between simple RT and forced choice. First, whereas inhibitory effects of exogenous cues at long SOAs are often found under simple RT, these same effects are difficult to observe under forced choice (see, e.g., Lupiáñez, Milán, Tornay, Madrid, \& Tudela, 1997). Second, whereas spatial 
cues cause increases in the magnitude of both the P1 and $\mathrm{N} 1$ event-related brain potentials when the task requires a visual discrimination, they cause only the former effect when a simple-RT task is employed (e.g., Mangun \& Hillyard, 1991). Thus, it appears that the benefits of a valid cue at the level of visual discrimination, regardless of overall cue validity, more than outweigh the costs of a low-validity cue at the level of visual encoding. Therefore, negative effects of exogenous cues are not observed in forced choice tasks, even when large numbers of display locations are employed.

In summary, with regard to the question posed in the title of this article - namely, why does the effect of shortSOA exogenous cuing on simple RT depend on the number of display locations? - the answer, paradoxically, is that it does not; at least, not directly. Instead, manipulations of display size in these experiments are confounded with changes in the overall validity of the cues and with the probability that the previous cue was valid, and these two changes have separate and additive effects on short-SOA exogenous cuing. The specific loci of these two effects and their possible links with other effects will be the subjects of subsequent work.

\section{AUTHOR NOTE}

The authors thank Cathleen Moore, Bill Prinzmetal, Shaun Vecera, and the reviewers for their comments on this work. Correspondence should be addressed to J. T. Mordkoff, Department of Psychology, University of Iowa, E11 Seashore Hall, Iowa City, IA 52242 (e-mail: jonathan-mordkoff@uiowa.edu).

\section{REFERENCES}

Boring, E. G. (1950). A history of experimental psychology. New York: Appleton-Century-Crofts.

CARrasco, M., \& Yeshurun, Y. (1998). The contribution of covert attention to the set-size and eccentricity effects in visual search. Journal of Experimental Psychology: Human Perception \& Performance, 24, 673-692.

Chen, P., Moore, C. M., \& Mordkoff, J. T. (2008). On the spatial metric of short-SOA costs of exogenous cuing. American Journal of Psychology, 121, 209-228.

Dodd, M. D., \& PratT, J. (2007). The effect of previous trial type on inhibition of return. Psychological Research, 71, 411-417.

Donders, F. C. (1969). On the speed of mental processes (W. G. Koster, Trans.). Acta Psychologica, 30, 412-431. (Original work published 1868)

Folk, C. L., Remington, R. W., \& Johnston, J. C. (1992). Involuntary covert orienting is contingent on attentional control settings. Journal of Experimental Psychology: Human Perception \& Performance, 18, 1030-1044

Frith, C. D., \& Done, D. J. (1986). Routes to action in reaction time tasks. Psychological Research, 48, 169-177.

Gawryszewski, L. G., Carreiro, L. R. R., \& Magalhães, F. V. (2005). Early and late inhibitions elicited by a peripheral visual cue on manual response to a visual target: Are they based on Cartesian coordinates? Psicológica, 26, 121-137.
Giray, M., \& UlRich, R. (1993). Motor coactivation revealed by response force in divided and focused attention. Journal of Experimental Psychology: Human Perception \& Performance, 19, 1278-1291.

KLEIN, R. M. (1980). Does oculomotor readiness mediate cognitive control of visual attention? In R. S. Nickerson (Ed.), Attention and performance VIII (pp. 259-276). Hillsdale, NJ: Erlbaum.

Luck, S. J., Hillyard, S. A., Mouloua, M., \& Hawkins, H. L. (1996). Mechanisms of visual-spatial attention: Resource allocation or uncertainty reduction? Journal of Experimental Psychology: Human Perception \& Performance, 22, 725-737.

Lupiáñez, J., Milán, E. G., Tornay, F. J., Madrid, E., \& Tudela, P. (1997). Does IOR occur in discrimination tasks? Yes, it does, but later. Perception \& Psychophysics, 59, 1241-1254.

Lupiáñez, J., Milliken, B., Solano, C., Weaver, B., \& Tipper, S. P. (2001). On the strategic modulation of the time course of facilitation and inhibition of return. Quarterly Journal of Experimental Psychology, 54A, 753-773.

Mangun, G. R., \& Hillyard, S. A. (1991). Modulations of sensoryevoked brain potentials indicate changes in perceptual processing during visual-spatial priming. Journal of Experimental Psychology: Human Perception \& Performance, 17, 1057-1074.

Maruff, P., Yucel, M., Danckert, J., Stuart, G., \& Currie, J. (1999). Facilitation and inhibition arising from the exogenous orienting of covert attention depends on the temporal properties of spatial cues and targets. Neuropsychologia, 37, 731-744.

McAuliffe, J., \& Pratt, J. (2005). The role of temporal and spatial factors in the covert orienting of visual attention tasks. Psychological Research, 69, 285-291.

Mordkoff, J. T., Miller, J., \& Roch, A.-C. (1996). Absence of coactivation in the motor component: Evidence from psychophysiological measures of target detection. Journal of Experimental Psychology: Human Perception \& Performance, 22, 25-41.

Müller, H. J., \& Findlay, J. M. (1988). The effect of visual attention on peripheral discrimination thresholds in single and multiple element displays. Acta Psychologica, 69, 129-155.

Posner, M. I., \& Cohen, Y. A. (1984). Components of visual orienting. In H. Bouma \& D. G. Bouwhuis (Eds.), Attention and performance $X$ (pp. 531-556). Hillsdale, NJ: Erlbaum.

Posner, M. I., Snyder, C. R. R., \& Davidson, B. J. (1980). Attention and the detection of signals. Journal of Experimental Psychology: General, 109, 160-174.

Pratt, J., Adam, J. J., \& McAuliffe, J. (1998). The spatial relationship between cues and targets mediates inhibition of return. Canadian Journal of Experimental Psychology, 52, 213-216.

PratT, J., Hillis, J., \& Gold, J. M. (2001). The effect of the physical characteristics of cues and targets on facilitation and inhibition. Psychonomic Bulletin \& Review, 8, 489-495.

Remington, R. W., Johnston, J. C., \& Yantis, S. (1992). Involuntary attentional capture by abrupt onsets. Perception \& Psychophysics, 51, 279-290.

Tassinari, G., Aglioti, S., Chelazzi, L., Peru, A., \& Berlucchi, G. (1994). Do peripheral non-informative cues induce early facilitation of target detection? Vision Research, 34, 179-189.

TheEUWES, J. (2004). Top-down search strategies cannot override attentional capture. Psychonomic Bulletin \& Review, 11, 65-70.

YANTIS, S. (1996). Attentional capture in vision. In A. F. Kramer, M. G. H. Coles, \& G. D. Logan (Eds.), Converging operations in the study of selective visual attention (pp. 45-76). Washington, DC: American Psychological Association.

(Manuscript received September 1, 2006; revision accepted for publication March 10, 2008.) 